\title{
Aqueous Phase Behavior of Ultra-Long Polyoxyethylene Surfactants
}

\author{
Md. Khalid Hossain, Carlos Rodriguez and Hironobu Kunieda* \\ Graduate School of Environment and Information Sciences, Yokohama National University
}

(79-7 Tokiwadai, Hodogaya-ku, Yokohama 240-8501, JAPAN)

Edited by T. Inoue, Fukuoka Univ., and accepted September 1, 2003 (received for review July 29, 2003)

\begin{abstract}
The aqueous phase behavior of poly(oxyethylene) alkyl ethers $\left(\mathrm{C}_{m} \mathrm{EO}_{n}\right)$ having both ultra-long hydrophobic and hydrophilic chains $(m=30$ and $n=94$ and 42) was investigated. The HLB values of $\mathrm{C}_{30} \mathrm{EO}_{94}$ and $\mathrm{C}_{30} \mathrm{EO}_{42}$ are 18 and 16, respectively. In $\mathrm{C}_{30} \mathrm{EO}_{94}$ system, micellar solution phase $\left(\mathrm{W}_{m}\right)$, micellar cubic $\left(\mathrm{I}_{1}\right)$, hexagonal $\left(\mathrm{H}_{1}\right)$ and solid surfactant phases (S) are successively formed. The same sequence is observed for $\mathrm{C}_{30} \mathrm{EO}_{42}$ system, but the $\mathrm{I}_{1}$ phase shrinks and the $\mathrm{H}_{1}$ phase prevails over a wide range of composition. This phase sequence was compared to that of conventional poly(oxyethylene) surfactants and it was found that the surfactant layer curvature of aggregates becomes less positive in a long hydrocarbonchain surfactant system even if the HLB values are the same.
\end{abstract}

Key words: phase behavior, polyoxyethylene surfactant

\section{Introduction}

Polyoxyethylene surfactants are widely used as emulsifying agents and detergents, and their aqueous phase behavior and self-organization have been extensively investigated (1-8). With increasing the polyoxyethylene chain length, the surfactant layer curvature of self-assemblies in water changes from negative to positive. However, the effect of the lipophilic chain on the self-organization of these surfactants has not been completely clarified, since the hydrocarbon chain length of conventional surfactants is restricted to the $\mathrm{C}_{8^{-}}$ $\mathrm{C}_{20}$ range.

On the other hand, it has been found that the phase behavior of amphiphilic copolymers in water is similar to that of nonionic surfactants. The morphology of selforganized structures is highly dependent on the compositional parameter $f$, defined as the ratio of A-chain (hydrophilic) volume to the total A-B copolymer vol- ume. Hence, both hydrophilic and hydrophobic chains are responsible for the formation of aggregates. In the surfactant field, the HLB value is used for the evaluation of the hydrophilic-lipophilic properties of surfactants. The HLB value is essentially equivalent to $f$, but the effect of lipophilic chain is usually neglected, because, as mentioned before, it is limited to a narrow range of carbon numbers. Since conventional surfactants and copolymers are very different in size, the relationships between the aqueous behavior of these two kinds of amphiphiles are still a matter of discussion.

In this context, we investigated the phase behavior of $\mathrm{C}_{30}$ nonionic surfactants with molecular sizes in between conventional surfactants and amphiphilic copolymers, and the result is compared with the phase behavior of conventional surfactant systems.

\footnotetext{
${ }^{*}$ Correspondence to: Hironobu KunIEDA, Graduate School of Environment and Information Sciences, Yokohama National University, 79-7

Tokiwadai, Hodogaya-ku, Yokohama 240-8501, JAPAN

E-mail: kunieda@ynu.ac.jp
} 


\section{Experimental}

\subsection{Materials}

Two polyoxyethylene-type nonionic surfactants, Unithox 480 and Unithox 490 were kindly supplied by Toyo Petrolite Co. They are designated as $\mathrm{C}_{30} \mathrm{EO}_{42}$ and $\mathrm{C}_{30} \mathrm{EO}_{94}$, respectively, and their properties are shown in Table 1. The manufacturer claims that the purities of Unithox 480 and 490 are about 95\% and 98\%, respectively. The main impurity is unreacted poly(ethylene).

Taking into account that the molar volumes of the oxyethylene group and the hydroxyl group are $38.8 \mathrm{~cm}^{3}$ $\mathrm{mol}^{-1}$ and $8.8 \mathrm{~cm}^{3} \mathrm{~mol}^{-1}$, respectively (9), the volume fraction of the hydrophilic part in total amphiphile, $f$, is estimated to be 0.77 for $\mathrm{C}_{30} \mathrm{EO}_{42}$ and 0.88 for $\mathrm{C}_{30} \mathrm{EO}_{94}$, respectively. The HLB values are approximately 16 and 18 , respectively $(\mathrm{HLB}=20 f)$.

Millipore filtered water was used to prepare the samples.

\subsection{Determination of Phase Diagrams}

The samples were prepared by weighing the appropriate amounts of the components in glass ampoules. The ampoules were flame-sealed immediately and the sample mixtures were homogenized using a vortex device. Viscous samples were homogenized further by repeated centrifugation in both directions through a narrow constriction to facilitate mixing. Phases were mainly identified by direct visual inspection and by observing the samples through cross polarizers. For a more precise identification of the liquid crystal phases, smallangle X-ray scattering (SAXS) measurements were carried out at $25^{\circ} \mathrm{C}$ on a small-angle scattering goniometer equipped with an $18 \mathrm{~kW}$ rotating anode (RINT-2500, Rigaku, Tokyo). Liquid crystal samples were covered by plastic films for the measurement (Mylar seal method).

\section{Results and Discussion}

\section{1 Phase Behavior of $\mathrm{C}_{30} \mathrm{EO}_{n}$ in Water}

The phase diagrams of binary water $/ \mathrm{C}_{30} \mathrm{EO}_{n}(n=42$ and 94) systems as a function of temperature are shown in Fig. 1.

In $\mathrm{C}_{30} \mathrm{EO}_{94}$ system, the aqueous micellar solution $\left(\mathrm{W}_{m}\right)$ phase is formed in the dilute region. As surfactant concentration increases, two types of liquid crystals, micellar cubic $\left(\mathrm{I}_{1}\right)$ and hexagonal $\left(\mathrm{H}_{1}\right)$ phases, are successively formed, and then the $\mathrm{H}_{1}$ phase changes into a solid phase or a surfactant liquid phase. The melting point of dry surfactant is close to the melting point of the polyoxyethylene chain. Usually, the phase diagrams of long alkyl chain surfactants show a wide solid region at room temperature (high Krafft point), but in the case of $\mathrm{C}_{30} \mathrm{EO}_{94}$ system, the melting temperature of solid surfactant decreases rapidly upon addition of water, probably due to the hydration of the very long polyoxyethylene chain. The maximum melting point of $\mathrm{C}_{30} \mathrm{EO}_{94}$ liquid crystals are approximately $100^{\circ} \mathrm{C}$ for the $\mathrm{I}_{1}$ phase and $55^{\circ} \mathrm{C}$ for the $\mathrm{H}_{1}$ phase, respectively. It is worth pointing out that for conventional polyoxyethylene type surfactants micellar cubic phases seldom exist at $100^{\circ} \mathrm{C}$, except for surfactants having bulky, rigid lipophilic groups such as cholesterol (11). It has been found that as the micellar hydrocarbon core gets larger, the thermal stability of the $I_{1}$ phase increases (12). The ratios of diffraction peaks of the $\mathrm{I}_{1}$ phase in $\mathrm{C}_{30} \mathrm{EO}_{94}$ aqueous system (Fig. 2) correspond to a body-centered cubic structure, which has been found in other non-ionic surfactant systems (12).

At very high temperatures and in the dilute region, phase separation associated with the cloud point takes place at around $110^{\circ} \mathrm{C}$.

When EO-chain length decreases from $\mathrm{C}_{30} \mathrm{EO}_{94}$ to $\mathrm{C}_{30} \mathrm{EO}_{42}$ (Fig. 1b), the $\mathrm{I}_{1}$ phase region shrinks and the $\mathrm{H}_{1}$ phase prevails over a wider range of composition. The maximum melting temperature of $\mathrm{H}_{1}$ phase is about

Table 1 Physical Properties of Surfactants.

\begin{tabular}{|c|c|c|c|}
\hline Surfactant & Molecular Formula & $\begin{array}{c}\text { Nominal Molecular } \\
\text { Weight }{ }^{1)}\end{array}$ & $\begin{array}{c}\text { Molar volume } \\
\left(\mathrm{cm}^{3} \mathrm{~mol}^{-1}\right)\end{array}$ \\
\hline $\mathrm{C}_{30} \mathrm{EO}_{42}$ & $\mathrm{CH}_{3}\left(\mathrm{CH}_{2}\right)_{29}\left(\mathrm{OCH}_{2} \mathrm{CH}_{2}\right)_{42} \mathrm{OH}$ & 2300 & 2141 \\
\hline $\mathrm{C}_{30} \mathrm{EO}_{94}$ & $\mathrm{CH}_{3}\left(\mathrm{CH}_{2}\right)_{29}\left(\mathrm{OCH}_{2} \mathrm{CH}_{2}\right)_{94} \mathrm{OH}$ & 4600 & 4159 \\
\hline
\end{tabular}

1) There is a distribution of polyoxyethylene chain lengths

2) Calculated using Tanford's equation (10) 
(a)

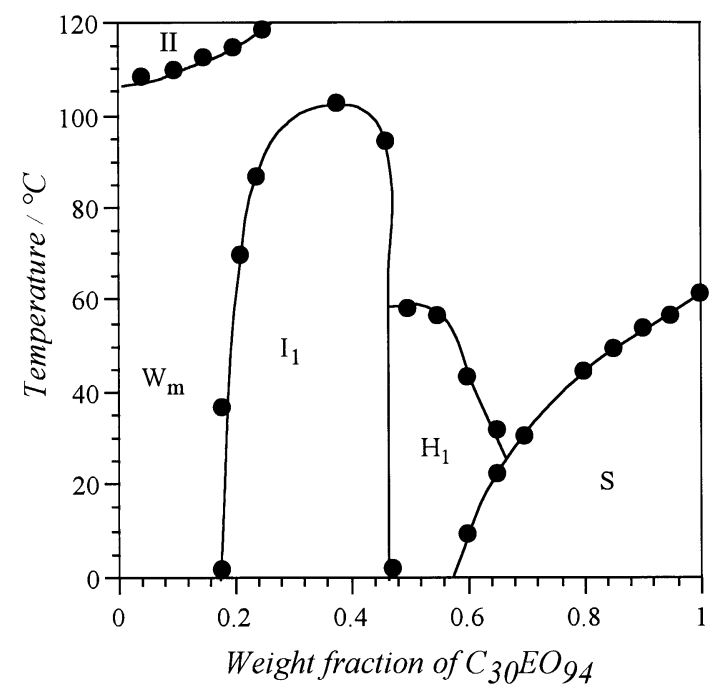

(b)

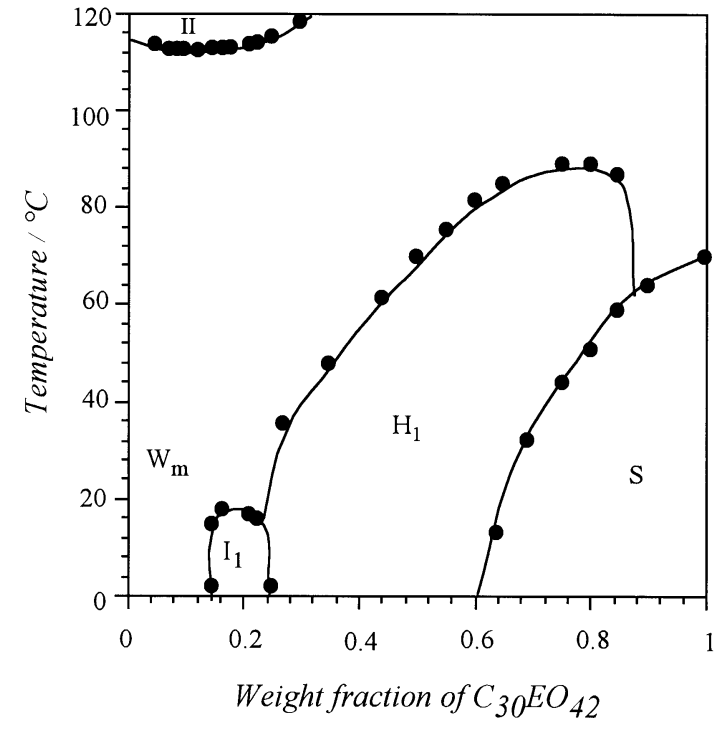

Fig. 1 Phase Diagram as a Function of the Weight Fraction of Surfactant.

(a) $\mathrm{C}_{30} \mathrm{EO}_{94}$-water system

(b) $\mathrm{C}_{30} \mathrm{EO}_{42}$-water system

II is a two-phase region.

$90^{\circ} \mathrm{C}$, which is relatively high for non-ionic surfactant systems. Similar to $\mathrm{C}_{30} \mathrm{EO}_{94}, \mathrm{C}_{30} \mathrm{EO}_{42}$ shows a low Krafft point. It is interesting that the cloud temperature of the less hydrophilic $\mathrm{C}_{30} \mathrm{EO}_{42}$ is slightly higher than that of $\mathrm{C}_{30} \mathrm{EO}_{94}$ system. As reported in a previous paper (12), the cloud temperature of polyoxyethylene-type surfactants approaches that of polyoxyethylene aqueous solutions when the EO chain is very long, therefore, there is a maximum in the plot of $\mathrm{C}_{m} \mathrm{EO}_{n}$ cloud temperature versus EO chain length.

\section{$3 \cdot 2$ Comparison with Other Nonionic Sur- factant Systems}

The morphology of surfactant self-organized structures are highly dependent on the surfactant layer curvature related to $f$ or HLB values as is shown in Fig. 3, which is reproduced from previous data $(8,13)$. With decreasing EO chain length or $f$ value, micellar cubic phase $\left(\mathrm{I}_{1}\right)$-hexagonal $\left(\mathrm{H}_{1}\right)$-lamellar $\left(\mathrm{L}_{\alpha}\right)$-reverse hexagonal $\left(\mathrm{H}_{2}\right)$ phase transition takes place. Therefore, by comparing the type of liquid crystal at a given $f$ or HLB value, we could understand the effect of hydrophobic chain length on the morphology of aggregates. The phase sequence as a function of surfactant concentration at $25^{\circ} \mathrm{C}$ for different polyoxyethylene-type surfactants is shown in Fig. 4. When the volume fraction of

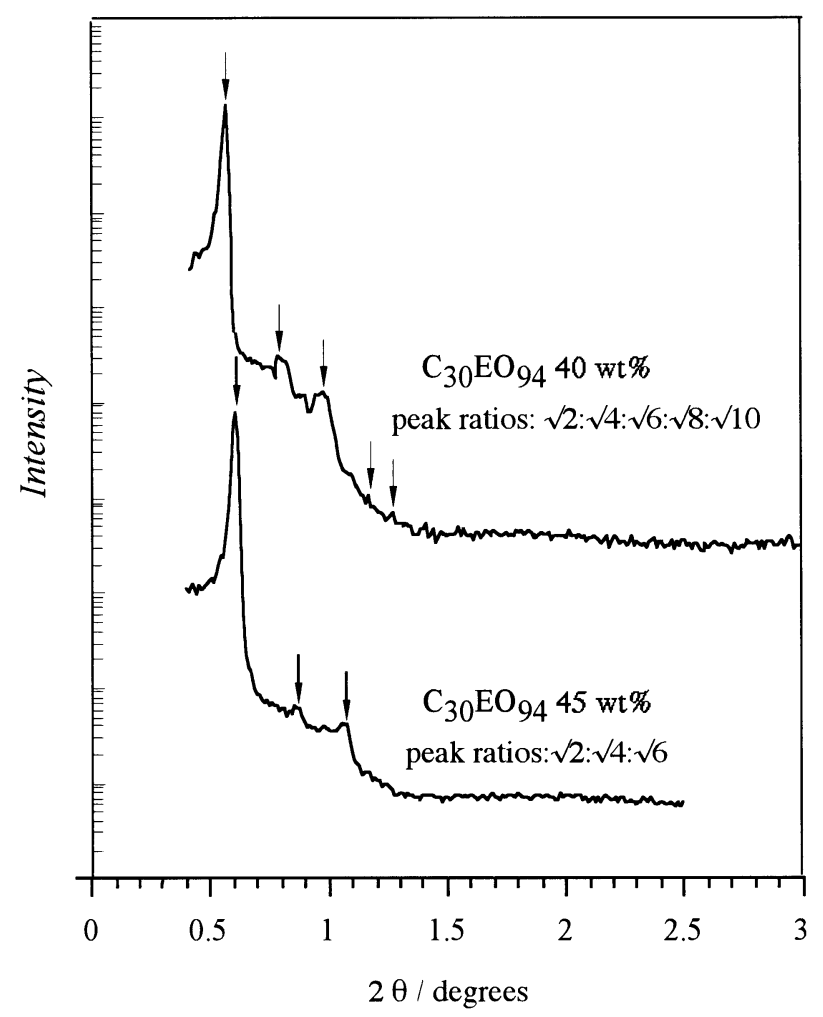

Fig. 2 Representative SAXS Spectra for the $\mathrm{I}_{1}$ Phase in $\mathrm{C}_{30} \mathrm{EO}_{94}$ Aqueous System. 
polyoxyethylene chain $f$ is around 0.77 (Fig. 4a), the $\mathrm{I}_{1}$ phase is present in all the systems except for $\mathrm{C}_{30} \mathrm{EO}_{42}$, which only forms the $\mathrm{H}_{1}$ phase in the liquid crystal region. It indicates that the curvature of $\mathrm{C}_{30} \mathrm{EO}_{42}$ aggregates is less positive than that of other polyoxyethylene surfactants having similar $f$, which can be attributed to the drastic change in the length of the hydrophobic chain.

When $f$ increases to around 0.88 (Fig. 4b), the $\mathrm{I}_{1}$ phase is the only liquid crystal phase found at room temperature, except for $\mathrm{C}_{30} \mathrm{EO}_{94}$ which forms both $\mathrm{I}_{1}$ and $\mathrm{H}_{1}$ phases. Again, this suggests that aggregates in $\mathrm{C}_{30} \mathrm{EO}_{94}$ system present a less positive curvature when compared to other systems even if the HLB value is similar. In polymer systems, it is well known that the

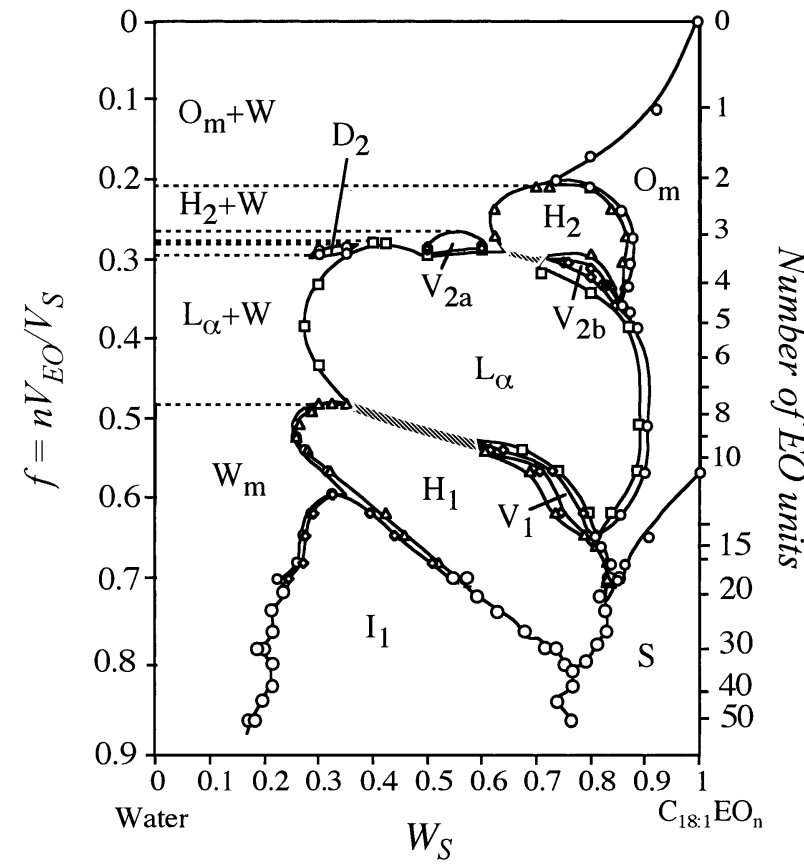

Fig. 3 Phase Diagram of the Water/ $\mathrm{C}_{18: 1} \mathrm{EO}_{n}$ Systems as a Function of the Weight Fraction of Surfactant, $\mathrm{W}_{\mathrm{S}}$ and the Volume Fraction of EO Chain in a Surfactant Molecule, $f=\mathrm{nV}_{\mathrm{E} 0} / \mathrm{V}_{\mathrm{S}}$ at $25^{\circ} \mathrm{C}$. The right-hand axis is the number of EO units per surfactant molecule. The notation is as follows: $\mathrm{W}_{m}$, micellar phase; $\mathrm{I}_{1}$, discontinuous cubic phase; $\mathrm{H}_{1}$, hexagonal phase; $\mathrm{V}_{1}$, bicontinuous cubic phase; $\mathrm{V}_{2}$, reverse bicontinuous cubic phase; $\mathrm{H}_{2}$, reverse hexagonal phase; $\mathrm{D}_{2}$, sponge phase; $\mathrm{L}_{\alpha}$, lamellar phase; $\mathrm{S}$, solid present phase; $\mathrm{O}_{m}$, surfactant liquid or reverse micellar phase. The Figure 3 is reproduced from the data in refs, 8 and 13 . effective chain length or the end to end distance is not proportional to polymerization degree or $m$, but $\sqrt{m}$ for a random flight-chain. Hence, the longer the chain, the more bulky it is. Namely, even if the HLB value is the same, the surfactant layer curvature becomes less posi-

(a)

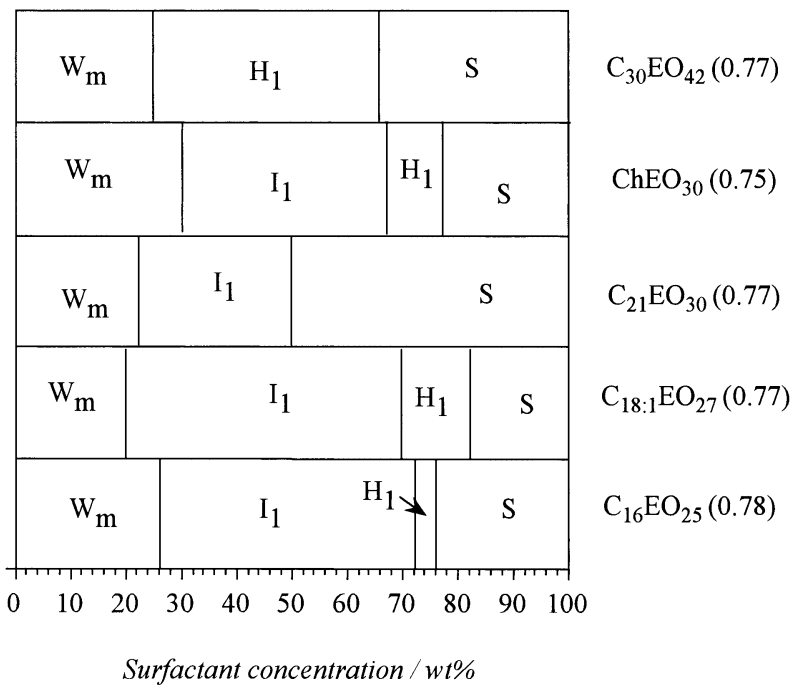

(b)

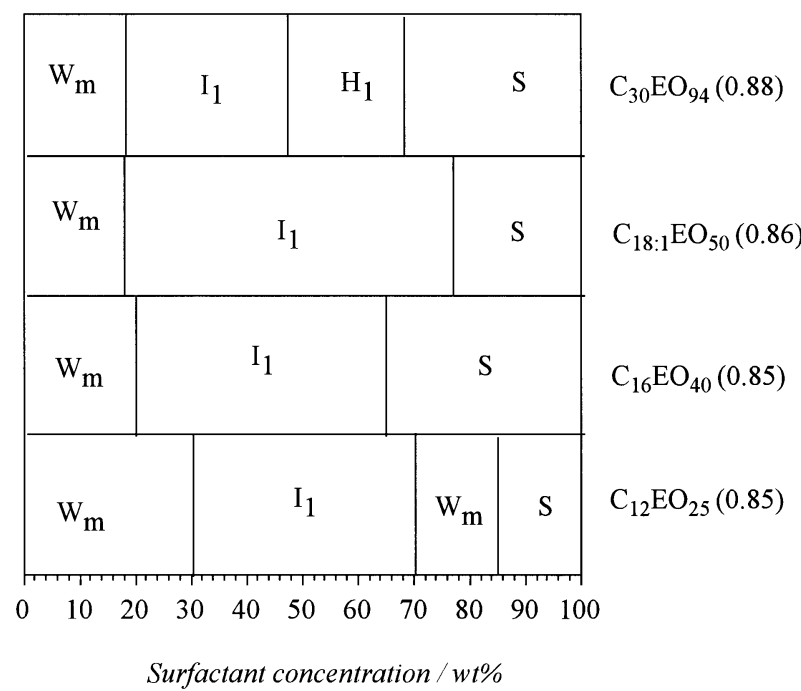

Fig. 4 Phase Sequences as a Function of Surfactant Concentration at $25^{\circ} \mathrm{C}$.

(a) $\mathrm{C}_{30} \mathrm{EO}_{42}$ and other nonionic systems with similar value of $f$. "Ch" indicates a cholesteryl group (b) $\mathrm{C}_{30} \mathrm{EO}_{94}$ and other nonionic systems with similar value of $f$. The actual value of $f$ is shown between brackets. The data are adapted from Figure 1 and from Refs. 11-13 
tive due to the bulky hydrocarbon chain (14). This effect is not remarkable for $\mathrm{C}_{8}-\mathrm{C}_{20}$ conventional surfactants, but the present $\mathrm{C}_{30}$ surfactant appears to be more lipophilic than expected from its HLB value. Hence, long $\mathrm{C}_{30} \mathrm{EO}_{n}$ surfactants behave as copolymers.

\section{References}

1. J.S. CLUNIE, J.F. GOODMAN and P.C. SYMONS, Phase Equilibriums of Hexaethyleneglycol Monododecyl Ether in Water, $J$. Chem Soc. Trans. Faraday Soc., Vol. 65, 287-296 (1969).

2. K. SHINODA, Thermodynamic Aspects of Nonionic SurfactantWater Systems, J. Colloid Interface Sci., Vol. 34, 278-282 (1970).

3. H. SAITO, Solution Properties of Nonionic Surfactant Aqueous Solutions and Their Relations with Foam Property, Nippon Kagaku Zasshi, Vol. 92, 223-226 (1971).

4. J.C. LANG and R.D. MORGAN, Nonionic Surfactant Mixtures. I. Phase Equilibria in $\mathrm{C}_{10} \mathrm{E}_{4}-\mathrm{H}_{2} \mathrm{O}$ and Closed-Loop Coexistence, J. Chem. Phys., Vol. 73, 5849-5861 (1980).

5. D.J. MITCHELL, G.F.T. TIDDY, L. WARING, T. BOSTOCK and M.P. McDONALD, Phase Behavior of Polyoxyethylene Surfactants with Water Mesophase Structures and Partial Miscibility (cloud points), J. Chem. Soc. Faraday Trans., Vol. 79, 975-1000 (1983).

6. R. STREY, R. SCHOMACKER, D. ROUX, F. NALLET and U.
OLSSON, Dilute Lamellar and $\mathrm{L}_{3}$ Phases in the Binary Water$\mathrm{C}_{12} \mathrm{E}_{5}$ System, J. Chem. Soc. Faraday Trans., Vol. 86, 2253-2261 (1990).

7. K. SHIGETA, M. SUZUKI and H. KUNIEDA, Phase Behavior of Polyoxyethylene Oleyl Ether in Water, Progr. Coll. Polym. Sci., 49-52 (1997).

8. H. KUNIEDA, K. SHIGETA, K. OZAWA and M. SUZUKI, Self-Organizing Structures in Poly(oxyethylene) Oleyl EtherWater System, J. Phys. Chem. B, Vol. 101, $7952-7957$ (1997).

9. K.-L. HUANG, K. SHIGETA and H. KUNIEDA, Phase Behavior of Polyoxyethylene Dodecyl Ether-Water Systems, Progr. Colloid Polym. Sci., Vol. 110, 171-174 (1998).

10. C. TANFORD, Micelle Shape and Size, J. Phys. Chem., Vol. 76, 3020-3024 (1972).

11. Y. TAMURA, Solubilization of Perfumes in Cubic Phases, Master Thesis, Yokohama National University (2000).

12. C. RODRIGUEZ, K. SHIGETA and H. KUNIEDA, CubicPhase-Based Concentrated Emulsions, J. Colloid Interface Sci., Vol. 223, 197-204 (2000).

13. K. SHIGETA, U. OLSSON and H. KUNIEDA, Correlation between Micellar Structure and Cloud Point in Long Poly(oxyethylene) Oleyl Ether Systems, Langmuir, Vol. 17, 4717-4723 (2001).

14. H. KUNIEDA, Md. H. UDDIN, M. HORII, H. FURUKAWA and A. HARASHIMA, Effect of Hydrophilic- and HydrophobicChain Lengths on the Phase Behavior of A-B-Type Silicone Surfactants in Water, J. Phys. Chem. B, Vol. 105, 5419-5426 (2001). 